\title{
On the closure of the Lizorkin space in spaces of Beppo Levi type
}

\author{
by \\ TAKAhide Kurokawa (Kagoshima) \\ Dedicated to Professor Masayuki Ito \\ on the occasion of his sixtieth birthday
}

\begin{abstract}
The purpose of this paper is to give a characterization of the closure of the Lizorkin space in spaces of Beppo Levi type. As preparations for the proof, we establish the invariance of the Lizorkin space, and give local integral representations for smooth functions.
\end{abstract}

1. Introduction and preliminaries. The purpose of this paper is to give a characterization of the closure of the Lizorkin space in spaces of Beppo Levi type. Let $\mathbb{R}^{n}$ be the $n$-dimensional Euclidean space. We denote the Schwartz space on $\mathbb{R}^{n}$ by $\mathcal{S}$. That is, $\mathcal{S}$ is the class of all $C^{\infty}$-functions $\varphi$ in $\mathbb{R}^{n}$ such that

$$
\sup _{x \in \mathbb{R}^{n}}\left|x^{\alpha} D^{\beta} \varphi(x)\right|<\infty
$$

for all multi-indices $\alpha=\left(\alpha_{1}, \ldots, \alpha_{n}\right)$ and $\beta=\left(\beta_{1}, \ldots, \beta_{n}\right)$ where $x^{\alpha}=$ $x_{1}^{\alpha_{1}} \ldots x_{n}^{\alpha_{n}}$ and $D^{\beta}=D_{1}^{\beta_{1}} \ldots D_{n}^{\beta_{n}}\left(D_{j}=\partial / \partial x_{j}\right)$. Unless otherwise specified, all functions are assumed to be complex-valued. The Schwartz space $\mathcal{S}$ contains the space $\mathcal{D}$ of all $C^{\infty}$-functions with compact support. The Lizorkin space $\Phi$ is defined by

$$
\Phi=\left\{\varphi \in \mathcal{S}: \int_{\mathbb{R}^{n}} \varphi(x) x^{\alpha} d x=0 \text { for all } \alpha\right\}
$$

([SKM: $§ 25$ in Chap. 5]). Further, we introduce the space $\Psi$ as follows:

$$
\Psi=\left\{\psi \in \mathcal{S}: D^{\alpha} \psi(0)=0 \text { for all } \alpha\right\} .
$$

2000 Mathematics Subject Classification: 31B99, $46 \mathrm{E} 35$.

Key words and phrases: Lizorkin space, spaces of Beppo Levi type, integral representations. 
The Fourier transform $\mathcal{F} u$ and the inverse Fourier transform $\overline{\mathcal{F}} u$ of an integrable function $u$ are defined by

$$
\mathcal{F} u(x)=\int e^{-i x \cdot y} u(y) d y, \quad \overline{\mathcal{F}} u(x)=\int e^{i x \cdot y} u(y) d y=\mathcal{F} u(-x)
$$

where $x \cdot y=x_{1} y_{1}+\ldots+x_{n} y_{n}$. By the Fourier inversion theorem, for $u \in \mathcal{S}$ we have the equality

$$
\overline{\mathcal{F}} \mathcal{F} u=\mathcal{F} \overline{\mathcal{F}} u=(2 \pi)^{n} u
$$

Noting that

$$
D^{\alpha}(\overline{\mathcal{F}} \varphi)(0)=\int \varphi(y)(i y)^{\alpha} d y
$$

and

$$
\int \mathcal{F} \psi(y)(i y)^{\alpha} d y=(2 \pi)^{n} D^{\alpha} \psi(0)
$$

for $\varphi, \psi \in \mathcal{S}$, we see that

$$
\Phi=\mathcal{F}(\Psi), \quad \Psi=\mathcal{F}(\Phi)
$$

by (1.1). Therefore, since the function $e^{-a|x|^{2}-b /|x|^{2}}(a>0, b>0)$ belongs to $\Psi$, the function $\mathcal{F}\left(e^{-a|x|^{2}-b /|x|^{2}}\right)$ is an example of a function in $\Phi$. We note that

$$
\Phi \cap \mathcal{D}=\{0\} .
$$

Throughout this paper, let $1<p<\infty$. For real numbers $r$ and $s$, we define the weighted Lebesgue spaces $L^{p, r,(\log )^{s}}$ as follows:

$$
\begin{aligned}
& L^{p, r,(\log )^{s}} \\
& \quad=\left\{u:\|u\|_{p, r,(\log )^{s}}=\left(\int|u(x)|^{p}(1+|x|)^{r p}(\log (e+|x|))^{s p} d x\right)^{1 / p}<\infty\right\}
\end{aligned}
$$

where $e$ is the base of the natural logarithm. We simply write $L^{p, 0,(\log )^{0}}=L^{p}$ and $\|u\|_{p, 0,(\log )^{0}}=\|u\|_{p}$. Further, $L^{1}$ denotes the space of all integrable functions, and $L_{\mathrm{loc}}^{1}$ is the set of all locally integrable functions. For a positive integer $m$, the Sobolev space $W_{m}^{p}$ and the space $\mathcal{L}_{m}^{p}$ of Beppo Levi type are defined by

$$
W_{m}^{p}=\left\{u \in L_{\mathrm{loc}}^{1}:\|u\|_{W_{m}^{p}}=\sum_{|\gamma| \leq m}\left\|D^{\gamma} u\right\|_{p}<\infty\right\}
$$

and

$$
\mathcal{L}_{m}^{p}=\left\{u \in L_{\mathrm{loc}}^{1}:|u|_{m, p}=\sum_{|\gamma|=m}\left\|D^{\gamma} u\right\|_{p}<\infty\right\}
$$

where $|\gamma|=\gamma_{1}+\ldots+\gamma_{n}$ for a multi-index $\gamma=\left(\gamma_{1}, \ldots, \gamma_{n}\right)$. The norm $\|u\|_{\mathcal{L}_{m}^{p}}$ in $\mathcal{L}_{m}^{p}$ is given by

$$
\|u\|_{\mathcal{L}_{m}^{p}}=\int_{|x|<1}|u(x)| d x+|u|_{m, p}
$$


The denseness of $\Phi$ in $L^{p}$ was established in P. I. Lizorkin [Li1] and S. G. Samko [Sa]. The denseness of $\Phi$ in the Bessel potential spaces is proved in P. I. Lizorkin [Li2], and hence $\Phi$ is dense in the Sobolev spaces $W_{m}^{p}$. In the final Section 4 , we give a characterization of the closure of $\Phi$ in spaces $\mathcal{L}_{m}^{p}$ of Beppo Levi type (Theorem 4.8). As a corollary we show that if $p \geq n$, then $\Phi$ is dense in $\mathcal{L}_{m}^{p}$, and if $p<n$, then $\Phi$ is not dense in $\mathcal{L}_{m}^{p}$ (Corollary 4.9). Sections 2 and 3 are preparations for Section 4. In Section 2 we establish the invariance of $\Phi$. Section 3 is devoted to local integral representations of smooth functions and interpolation inequalities. Throughout this paper we use the symbol $C$ for a generic positive constant whose value may be different at each occurrence.

2. The invariance of $\Phi$. In this section we establish the invariance of $\Phi$ relative to a class of operators which contains differential operators, the Riesz transforms and the Riesz potential operators. Let $\mathbb{N}$ denote the set of natural numbers including zero; $2 \mathbb{N}$ stands for the set of nonnegative even numbers.

Lemma 2.1. Let $m \in \mathbb{N}$. If

$$
\lim _{|x| \rightarrow 0} \frac{1}{|x|^{m}} \sum_{|\gamma|=m} a_{\gamma} x^{\gamma}=0,
$$

then $a_{\gamma}=0$ for all $\gamma$ with $|\gamma|=m$.

Proof. We put $P(x)=\sum_{|\gamma|=m} a_{\gamma} x^{\gamma}$. By assumption, for $\Theta$ with $|\Theta|=1$ we have

$$
0=\lim _{t \rightarrow 0+0} \frac{P(t \Theta)}{|t \Theta|^{m}}=\lim _{t \rightarrow 0+0} \frac{t^{m} P(\Theta)}{t^{m}}=P(\Theta) .
$$

For $x \neq 0$, by putting $t=|x|$ and $\Theta=x /|x|$, we get

$$
P(x)=P(t \Theta)=t^{m} P(\Theta)=0 .
$$

Since it is clear that $P(0)=0$, we conclude that $P(x)$ is identically 0 , and hence $a_{\gamma}=0$ for all $\gamma$ with $|\gamma|=m$.

We define

$$
\mathcal{H}=\left\{f \in C^{\infty}\left(\mathbb{R}^{n}\right): D^{\alpha} f(0)=0 \text { for all } \alpha\right\} .
$$

Lemma 2.2. Let $f \in C^{\infty}\left(\mathbb{R}^{n}\right)$. Then the following three conditions are equivalent:

(I) $f \in \mathcal{H}$.

(II) For any multi-index $\alpha$ and any $l \in \mathbb{N}$,

$$
\lim _{|x| \rightarrow 0} \frac{D^{\alpha} f(x)}{|x|^{l}}=0 .
$$


(III) For any $l \in \mathbb{N}$,

$$
\lim _{|x| \rightarrow 0} \frac{f(x)}{|x|^{l}}=0 .
$$

Proof. First, we show (I) $\Rightarrow($ II). Let $f \in \mathcal{H}$. For any $l \in \mathbb{N}$, by the condition $f \in \mathcal{H}$ and Taylor's formula, we have

$$
f(x)=\sum_{|\gamma|=l} \frac{D^{\gamma} f(\theta x)}{\gamma !} x^{\gamma}, \quad 0<\theta<1 .
$$

Hence

$$
\lim _{|x| \rightarrow 0} \frac{f(x)}{|x|^{l}}=\sum_{|\gamma|=l} \lim _{|x| \rightarrow 0} \frac{D^{\gamma} f(\theta x)}{\gamma !} \cdot \frac{x^{\gamma}}{|x|^{l}}=0,
$$

because $\lim _{|x| \rightarrow 0} D^{\gamma} f(\theta x)=D^{\gamma} f(0)=0$. Moreover, since $D^{\alpha} f \in \mathcal{H}$ for any multi-index $\alpha$, by applying the above argument to $D^{\alpha} f$, we get

$$
\lim _{|x| \rightarrow 0} \frac{D^{\alpha} f(x)}{|x|^{l}}=0
$$

for any $l \in \mathbb{N}$. Thus we obtain (II). The implication (II) $\Rightarrow($ III) is trivial.

We show $(\mathrm{III}) \Rightarrow(\mathrm{I})$. First, by taking $l=0$, we get $f(0)=\lim _{|x| \rightarrow 0} f(x)$ $=0$. We assume that $D^{\gamma} f(0)=0$ for any $\gamma$ with $|\gamma| \leq m$. Then by Taylor's formula we have

$\frac{f(x)}{|x|^{m+1}}=\frac{1}{|x|^{m+1}} \sum_{|\gamma|=m+1} \frac{D^{\gamma} f(0)}{\gamma !} x^{\gamma}+\sum_{|\gamma|=m+2} \frac{D^{\gamma} f(\theta x)}{\gamma !} \cdot \frac{x^{\gamma}}{|x|^{m+1}}, \quad 0<\theta<1$.

By assumption we have $\lim _{|x| \rightarrow 0} f(x) /|x|^{m+1}=0$, and moreover for $\gamma$ with $|\gamma|=m+2, \lim _{|x| \rightarrow 0} x^{\gamma} /|x|^{m+1}=0$. Therefore

$$
\lim _{|x| \rightarrow 0} \frac{1}{|x|^{m+1}} \sum_{|\gamma|=m+1} \frac{D^{\gamma} f(0)}{\gamma !} x^{\gamma}=0 .
$$

Hence, it follows from Lemma 2.1 that $D^{\gamma} f(0)=0$ for any $\gamma$ with $|\gamma|=m+1$. Consequently, by induction we obtain $D^{\gamma} f(0)=0$ for any $\gamma$, and hence $f \in \mathcal{H}$. This proves $(\mathrm{III}) \Rightarrow(\mathrm{I})$.

Let $\Omega \subset \mathbb{R}^{n}$ be an open set and $m \in \mathbb{N}$. $C^{m}(\Omega)$ is the space of functions such that all partial derivatives $D^{\alpha} f,|\alpha| \leq m$, are continuous on $\Omega$, and $C^{\infty}(\Omega)=\bigcap_{m=0}^{\infty} C^{m}(\Omega)$. The following lemma is a consequence of the mean value theorem of calculus.

Lemma 2.3. Let $f \in C^{0}\left(\mathbb{R}^{n}\right)$ and $f \in C^{\infty}\left(\mathbb{R}^{n}-\{0\}\right)$. If $\lim _{|x| \rightarrow 0} D^{\alpha} f(x)$ exists for any multi-index $\alpha$, then $f \in C^{\infty}\left(\mathbb{R}^{n}\right)$.

Lemma 2.4. Let $\psi \in \Psi$ and $m \in C^{\infty}\left(\mathbb{R}^{n}-\{0\}\right)$. If for any multi-index $\gamma$, there exist $l_{\gamma} \in \mathbb{N}$ and $C_{\gamma}>0$ such that 


$$
\left|D^{\gamma} m(x)\right| \leq C_{\gamma} \begin{cases}|x|^{l_{\gamma}}, & |x| \geq 1, \\ |x|^{-l_{\gamma}}, & 0<|x|<1,\end{cases}
$$

then the function

$$
f(x)= \begin{cases}m(x) \psi(x), & x \neq 0 \\ 0, & x=0\end{cases}
$$

is in $\Psi$.

Proof. First, it is clear that $f \in C^{\infty}\left(\mathbb{R}^{n}-\{0\}\right)$. By (2.1), the assumption $\psi \in \Psi$ and Lemma 2.2 we see that

$$
\lim _{|x| \rightarrow 0}|f(x)|=\lim _{|x| \rightarrow 0}|m(x) \psi(x)| \leq C_{0} \lim _{|x| \rightarrow 0} \frac{|\psi(x)|}{|x|^{l_{0}}}=0,
$$

and hence $f \in C^{0}\left(\mathbb{R}^{n}\right)$. We show

$$
\lim _{|x| \rightarrow 0} D^{\alpha} f(x)=0 \quad \text { for any } \alpha .
$$

Since

$$
D^{\alpha} f(x)=\sum_{\gamma \leq \alpha}\left(\begin{array}{l}
\alpha \\
\gamma
\end{array}\right) D^{\gamma} m(x) D^{\alpha-\gamma} \psi(x)
$$

by the Leibniz formula, it is sufficient to show

$$
D^{\gamma} m(x) D^{\alpha-\gamma} \psi(x) \rightarrow 0 \quad(|x| \rightarrow 0)
$$

for $\gamma \leq \alpha$, which follows from (2.1), $\psi \in \Psi$ and Lemma 2.2 again. Hence we obtain (2.2), and hence $f \in C^{\infty}\left(\mathbb{R}^{n}\right)$ by Lemma 2.3. Further, (2.2) also implies $D^{\alpha} f(0)=0$ for any multi-index $\alpha$, and hence $f \in \mathcal{H}$. Similarly, the condition (2.1) and $\psi \in \Psi$ imply $f \in \mathcal{S}$. Thus we obtain $f \in \mathcal{H} \cap \mathcal{S}=\Psi$.

The dual space of $\mathcal{S}$ is denoted by $\mathcal{S}^{\prime}$.

Proposition 2.5. Suppose $T: \Phi \rightarrow \mathcal{S}^{\prime}$ is a linear operator, and for $\varphi \in \Phi$,

$$
\mathcal{F}(T \varphi)(x)=m(x) \mathcal{F} \varphi(x) \quad \text { in } \mathcal{S}^{\prime},
$$

where $m \in C^{\infty}\left(\mathbb{R}^{n}-\{0\}\right)$ and for any multi-index $\gamma$ there exist $l_{\gamma} \in \mathbb{N}$ and $C_{\gamma}>0$ such that

$$
\left|D^{\gamma} m(x)\right| \leq C_{\gamma} \begin{cases}|x|^{l_{\gamma}}, & |x| \geq 1 \\ |x|^{-l_{\gamma}}, & 0<|x|<1 .\end{cases}
$$

Then the operator $T$ carries $\Phi$ into $\Phi$.

Proof. For $\varphi \in \Phi$, by Lemma 2.4 the function

$$
\psi(x)= \begin{cases}m(x) \mathcal{F} \varphi(x), & x \neq 0, \\ 0, & x=0,\end{cases}
$$

is in $\Psi$ since $\mathcal{F} \varphi \in \Psi$. Therefore we have

$$
T \varphi=\overline{\mathcal{F}} \mathcal{F}(T \varphi)=\overline{\mathcal{F}} \psi \in \Phi .
$$


For a positive integer $m$, the Riesz kernel $\kappa_{m}(x)$ of order $m$ is given by

$$
\kappa_{m}(x)=\frac{1}{\gamma_{m, n}} \begin{cases}|x|^{m-n}, & m-n \notin 2 \mathbb{N}, \\ \left(\delta_{m, n}-\log |x|\right)|x|^{m-n}, & m-n \in 2 \mathbb{N},\end{cases}
$$

with

$$
\gamma_{m, n}= \begin{cases}\pi^{n / 2} 2^{m} \Gamma(m / 2) / \Gamma((n-m) / 2), & m-n \notin 2 \mathbb{N}, \\ (-1)^{(m-n) / 2} 2^{m-1} \pi^{n / 2} \Gamma(m / 2)((m-n) / 2) !, & m-n \in 2 \mathbb{N},\end{cases}
$$

and

$$
\delta_{m, n}=\frac{\Gamma^{\prime}(m / 2)}{2 \Gamma(m)}+\frac{1}{2}\left(1+\frac{1}{2}+\ldots+\frac{1}{(m-n) / 2}-\mathcal{C}\right)-\log \pi
$$

where $\mathcal{C}$ is Euler's constant. For $f \in \mathcal{S}$, we define the Riesz potential $\kappa_{m}^{f}$ of $f$ as follows:

$$
\kappa_{m}^{f}(x)=\int \kappa_{m}(x-y) f(y) d y .
$$

The Fourier transform of the Riesz kernel $\kappa_{m}$ is given by

$$
\mathcal{F} \kappa_{m}(x)=\text { Pf. }|x|^{-m} \quad([\text { Sc: Sect. } 7 \text { in Chap. VII }])
$$

where Pf. stands for the pseudo function ([Sc: Sect. 4 in Chap. II]). Since

$$
\text { Pf. }|x|^{-m} \psi(x)=|x|^{-m} \psi(x)
$$

for $\psi \in \Psi$, we have

$$
\mathcal{F} \kappa_{m}^{f}(x)=|x|^{-m} \mathcal{F} f(x)
$$

for $f \in \Phi$. Further, we define the Riesz transforms $R_{j}(j=1, \ldots, n)$ as follows:

$$
R_{j} f(x)=\lim _{\varepsilon \rightarrow 0} \frac{\Gamma((n+1) / 2)}{\pi^{(n+1) / 2}} \int_{|x-y| \geq \varepsilon} \frac{x_{j}-y_{j}}{|x-y|^{n+1}} f(y) d y .
$$

The Riesz transforms are bounded operators on $L^{p}$ :

$$
\left\|R_{j} f\right\|_{p} \leq C\|f\|_{p}, \quad j=1, \ldots, n,
$$

and for $f \in L^{2}$, the Fourier transform of $R_{j} f$ is given by

$$
\mathcal{F}\left(R_{j} f\right)(x)=-\frac{i x_{j}}{|x|} \mathcal{F} f(x) .
$$

For a multi-index $\gamma=\left(\gamma_{1}, \ldots, \gamma_{n}\right)$, we set

$$
R^{\gamma}=R_{1}^{\gamma_{1}} \ldots R_{n}^{\gamma_{n}} .
$$

For $f \in L^{2}$, it follows from (2.5) that

$$
\mathcal{F}\left(R^{\gamma} f\right)(x)=\frac{(-i)^{|\gamma|} x^{\gamma}}{|x|^{|\gamma|}} \mathcal{F} f(x) .
$$

COROLLARY 2.6. The differential operator $D^{\gamma}$, the Riesz potential operator $\kappa_{m}^{f}$ and the Riesz transforms $R_{j}(j=1, \ldots, n)$ carry $\Phi$ into $\Phi$. 
Proof. This follows from the formula $\mathcal{F}\left(D^{\gamma} f\right)(x)=(i x)^{\gamma} \mathcal{F} f(x),(2.3)$, (2.5) and Proposition 2.5.

REMARK 2.7. The invariance of $\Phi$ relative to the Riesz potential operator was proved in $[\mathrm{He}]$ and $[\mathrm{SKM}]$.

For $f \in \mathcal{L}_{m}^{p}$ we put

$$
D^{m} f=\sum_{|\gamma|=m} \frac{m !}{\gamma !} R^{\gamma} D^{\gamma} f .
$$

Corollary 2.8. If $u \in \Phi$, then $f=D^{m} u \in \Phi$ and $u=\kappa_{m}^{f}$.

Proof. That $D^{m} u \in \Phi$ follows from Corollary 2.6. Since

$$
\mathcal{F}\left(D^{m} u\right)(x)=|x|^{m} \mathcal{F} u(x)
$$

for $u \in \Phi$ by (2.6), we see that

$$
\begin{aligned}
\mathcal{F} \kappa_{m}^{f}(x) & =\mathcal{F} \kappa_{m}(x) \mathcal{F} f(x)=\mathcal{F} \kappa_{m}(x) \mathcal{F}\left(D^{m} u\right)(x) \\
& =\text { Pf. }|x|^{-m}|x|^{m} \mathcal{F} u(x)=\mathcal{F} u(x) .
\end{aligned}
$$

3. Local integral representations of smooth functions. If $u$ is a $C^{m}$-function with compact support, then it can be represented by its partial derivatives of order $m$ as follows:

$$
u(x)=\sum_{|\alpha|=m} \frac{m}{\alpha ! \sigma_{n}} \int \frac{(x-y)^{\alpha}}{|x-y|^{n}} D^{\alpha} u(y) d y
$$

([Re]) where $\sigma_{n}$ is the surface area of the unit sphere, and

$$
u(x)=\sum_{|\alpha|=m} \frac{(-1)^{m} m !}{\alpha !} \int D^{\alpha} \kappa_{2 m}(x-y) D^{\alpha} u(y) d y
$$

([Wa]). In this section we give two kinds of integral representations of $C^{m}$-functions, which correspond to (3.1) and (3.2). One is based on Taylor's formula, and the other is deduced from the fact that the Riesz kernel $\kappa_{2 m}$ is a fundamental solution for the iterated Laplace operator $\Delta^{m}$, namely

$$
\Delta^{m} \kappa_{2 m}=(-1)^{m} \delta
$$

where $\delta$ is the point mass at the origin.

Let $0<\varepsilon_{1}<\varepsilon_{2}$. We take a function $\eta \in C^{\infty}(\mathbb{R})$ such that $\operatorname{supp} \eta \subset$ $\left\{\varepsilon_{1} \leq t \leq \varepsilon_{2}\right\}$ and

$$
\int_{0}^{\infty} \eta(t) t^{n-1} d t=\frac{1}{\sigma_{n}}
$$

and set

$$
\tau(t)=\int_{t}^{\infty} \eta(s) s^{n-1} d s .
$$


Moreover we put

$$
\begin{aligned}
& \omega(x)=\eta(|x|), \\
& \chi(x)=\sigma_{n} \int_{|x|}^{\infty} \omega(t x /|x|) t^{n-1} d t .
\end{aligned}
$$

Then $\int \omega(x) d x=1$ and $\chi(x)=\sigma_{n} \tau(|x|)$. Since $\tau \in C^{\infty}([0, \infty))$ and

$$
\tau(t)= \begin{cases}1 / \sigma_{n} & \text { for } 0 \leq t \leq \varepsilon_{1} \\ 0 & \text { for } t \geq \varepsilon_{2}\end{cases}
$$

we have $\chi \in \mathcal{D}$ and

$$
\chi(x)= \begin{cases}1 & \text { for }|x| \leq \varepsilon_{1} \\ 0 & \text { for }|x| \geq \varepsilon_{2} .\end{cases}
$$

Proposition 3.1. Let $0<\varepsilon_{1}<\varepsilon_{2}$. Then there exist functions $\mu, \chi \in \mathcal{D}$ such that $\operatorname{supp} \mu, \operatorname{supp} \chi \subset\left\{|x| \leq \varepsilon_{2}\right\}, \mu(x)=0$ on $\left\{|x| \leq \varepsilon_{1}\right\}, \chi(x)=1$ on $\left\{|x| \leq \varepsilon_{1}\right\}$, and if $u \in C^{m}\left(\mathbb{R}^{n}\right)$, then

$$
u(x)=\int \mu(x-y) u(y) d y+\sum_{|\alpha|=m} \frac{m}{\alpha ! \sigma_{n}} \int \frac{(x-y)^{\alpha}}{|x-y|^{n}} \chi(x-y) D^{\alpha} u(y) d y .
$$

Proof. By Taylor's formula we have

$$
\begin{aligned}
u(x)= & \sum_{|\gamma|<m} \frac{D^{\gamma} u(y)}{\gamma !}(x-y)^{\gamma} \\
& +m \sum_{|\alpha|=m} \frac{(x-y)^{\alpha}}{\alpha !} \int_{0}^{1}(1-t)^{m-1} D^{\alpha} u(y+t(x-y)) d t .
\end{aligned}
$$

We take functions $\omega$ and $\chi$ defined by (3.4) and (3.5). Multiplying (3.7) by $\omega(x-y)$ and integrating with respect to $y$, we get

$$
\begin{aligned}
u(x)= & \sum_{|\gamma|<m} \frac{1}{\gamma !} \int D^{\gamma} u(y)(x-y)^{\gamma} \omega(x-y) d y \\
& +\sum_{|\alpha|=m} \frac{m}{\alpha !} \int(x-y)^{\alpha} \omega(x-y) \int_{0}^{1}(1-t)^{m-1} D^{\alpha} u(y+t(x-y)) d t d y \\
= & \sum_{|\gamma|<m} \frac{1}{\gamma !} \int D^{\gamma} u(y)(x-y)^{\gamma} \omega(x-y) d y \\
& +\sum_{|\alpha|=m} \frac{m}{\alpha !} \int_{0}^{1}(1-t)^{m-1}\left(\int(x-y)^{\alpha} \omega(x-y) D^{\alpha} u(y+t(x-y)) d y\right) d t \\
= & I_{1}(x)+I_{2}(x) .
\end{aligned}
$$


By integration by parts we have

$$
I_{1}(x)=\int \mu(x-y) u(y) d y \quad \text { where } \quad \mu(x)=\sum_{|\gamma|<m} \frac{1}{\gamma !} D^{\gamma}\left(x^{\gamma} \omega(x)\right) .
$$

Since $\omega \in \mathcal{D}$ and $\operatorname{supp} \omega \subset\left\{\varepsilon_{1} \leq|x| \leq \varepsilon_{2}\right\}$ by (3.4), $\mu$ also has the same properties. Further by the change of variables $y+t(x-y)=z$, we obtain

$$
\begin{aligned}
I_{2}(x) & =\sum_{|\alpha|=m} \frac{m}{\alpha !} \int_{0}^{1}(1-t)^{m-1}\left(\int \frac{(x-z)^{\alpha}}{(1-t)^{m}} \omega\left(\frac{x-z}{1-t}\right) D^{\alpha} u(z) \frac{d z}{(1-t)^{n}}\right) d t \\
& =\sum_{|\alpha|=m} \frac{m}{\alpha !} \int D^{\alpha} u(z)(x-z)^{\alpha}\left(\int_{0}^{1} \omega\left(\frac{x-z}{1-t}\right) \frac{d t}{(1-t)^{n+1}}\right) d z
\end{aligned}
$$

because $x-y=(x-z) /(1-t)$. By the change of variable $|x-z| /(1-t)=s$, we get

$$
\begin{aligned}
I_{2}(x) & =\sum_{|\alpha|=m} \frac{m}{\alpha !} \int D^{\alpha} u(z)(x-z)^{\alpha}\left(\int_{|x-z|}^{\infty} \omega\left(s \frac{x-z}{|x-z|}\right) \frac{s^{n-1}}{|x-z|^{n}} d s\right) d z \\
& =\sum_{|\alpha|=m} \frac{m}{\alpha ! \sigma_{n}} \int D^{\alpha} u(z) \frac{(x-z)^{\alpha}}{|x-z|^{n}} \chi(x-z) d z
\end{aligned}
$$

because $(x-z) /(1-t)=s(x-z) /|x-z|$. Thus we obtain (3.6).

Proposition 3.2. Let $0<\varepsilon_{1}<\varepsilon_{2}$. Then there exist functions $\zeta, \xi \in \mathcal{D}$ such that $\operatorname{supp} \zeta, \operatorname{supp} \xi \subset\left\{|x| \leq \varepsilon_{2}\right\}, \zeta(x)=0$ on $\left\{|x| \leq \varepsilon_{1}\right\}, \xi(x)=1$ on $\left\{|x| \leq \varepsilon_{1}\right\}$, and if $u \in C^{m}\left(\mathbb{R}^{n}\right)$, then

$$
u(x)=\int \zeta(x-y) u(y) d y+\sum_{|\alpha|=m} \frac{(-1)^{m} m !}{\alpha !} \int D^{\alpha}\left(\xi \kappa_{2 m}\right)(x-y) D^{\alpha} u(y) d y
$$

Proof. First we assume that $u \in \mathcal{D}$. Since $u(x-y)$ belongs to $\mathcal{D}$ as a function of $y$, the formula (3.3) gives

$$
\begin{aligned}
u(x) & =\langle\delta(y), u(x-y)\rangle=\left\langle(-1)^{m} \Delta^{m} \kappa_{2 m}(y), u(x-y)\right\rangle \\
& =\left\langle(-1)^{m} \kappa_{2 m}(y), \Delta^{m} u(x-y)\right\rangle=\int(-1)^{m} \kappa_{2 m}(y) \Delta^{m} u(x-y) d y
\end{aligned}
$$

where $\langle\cdot, \cdot\rangle$ stands for the pairing between distributions and test functions. We take a function $\xi \in \mathcal{D}$ such that

$$
\xi(x)= \begin{cases}1, & |x| \leq \varepsilon_{1} \\ 0, & |x| \geq \varepsilon_{2}\end{cases}
$$

If we set $\zeta(x)=(-1)^{m} \Delta^{m}\left((1-\xi) \kappa_{2 m}\right)(x)$, then by integration by parts we 
have

$$
\begin{aligned}
u(x)= & \int(1-\xi(y))(-1)^{m} \kappa_{2 m}(y) \Delta^{m} u(x-y) d y \\
& +\int \xi(y)(-1)^{m} \kappa_{2 m}(y) \Delta^{m} u(x-y) d y \\
= & \int \zeta(y) u(x-y) d y+\sum_{|\alpha|=m} \frac{(-1)^{m} m !}{\alpha !} \int D^{\alpha}\left(\xi \kappa_{2 m}\right)(y) D^{\alpha} u(x-y) d y \\
= & \int \zeta(x-y) u(y) d y+\sum_{|\alpha|=m} \frac{(-1)^{m} m !}{\alpha !} \int D^{\alpha}\left(\xi \kappa_{2 m}\right)(x-y) D^{\alpha} u(y) d y .
\end{aligned}
$$

By (3.3) and (3.9) we see that $\zeta(x)=0$ for $|x| \leq \varepsilon_{1}$ and $|x| \geq \varepsilon_{2}$, and hence $\zeta \in \mathcal{D}$. Therefore we obtain the proposition for $u \in \mathcal{D}$. In case $u \in C^{m}\left(\mathbb{R}^{n}\right)$, the proposition is obtained by approximating $u$ by a sequence $\left\{u_{j}\right\} \subset \mathcal{D}$ such that $D^{\alpha} u_{j}$ converges to $D^{\alpha} u$ locally uniformly as $j \rightarrow \infty$ for $|\alpha| \leq m$. This completes the proof.

By taking differentiation under the integral sign in (3.6) and (3.8), we obtain the following corollary.

Corollary 3.3. Let $0<\varepsilon_{1}<\varepsilon_{2}$. Then there exist functions $\mu, \chi, \zeta, \xi \in \mathcal{D}$ such that $\operatorname{supp} \mu, \operatorname{supp} \chi, \operatorname{supp} \zeta, \operatorname{supp} \xi \subset\left\{|x| \leq \varepsilon_{2}\right\}, \mu(x)=\zeta(x)=0$ on $\left\{|x| \leq \varepsilon_{1}\right\}, \chi(x)=\xi(x)=1$ on $\left\{|x| \leq \varepsilon_{1}\right\}$, and if $u \in C^{m}\left(\mathbb{R}^{n}\right)$, then for $|\gamma| \leq m-1$

$$
D^{\gamma} u(x)=\int D^{\gamma} \mu(x-y) u(y) d y+\sum_{|\alpha|=m} \frac{m}{\alpha ! \sigma_{n}} \int D^{\gamma} \chi_{\alpha}(x-y) D^{\alpha} u(y) d y
$$

where $\chi_{\alpha}(x)=x^{\alpha} \chi(x) /|x|^{n}$, and

$$
\begin{aligned}
D^{\gamma} u(x)= & \int D^{\gamma} \zeta(x-y) u(y) d y \\
& +\sum_{|\alpha|=m} \frac{(-1)^{m} m !}{\alpha !} \int D^{\gamma+\alpha}\left(\xi \kappa_{2 m}\right)(x-y) D^{\alpha} u(y) d y .
\end{aligned}
$$

We set $B_{1}=\{|x|<1\}$. By cutting off functions belonging to $C^{m}\left(B_{1}\right)$, we obtain integral representations for $u \in C^{m}\left(B_{1}\right)$.

COROllary 3.4. Let $r>0,0<\varepsilon_{1}<\varepsilon_{2}$ and $r+\varepsilon_{2}<1$. Then there exist functions $\mu, \chi, \zeta, \xi \in \mathcal{D}$ such that $\operatorname{supp} \mu, \operatorname{supp} \chi, \operatorname{supp} \zeta, \operatorname{supp} \xi \subset\left\{|x| \leq \varepsilon_{2}\right\}$, $\mu(x)=\zeta(x)=0$ on $\left\{|x| \leq \varepsilon_{1}\right\}, \chi(x)=\xi(x)=1$ on $\left\{|x| \leq \varepsilon_{1}\right\}$, and if $u \in C^{m}\left(B_{1}\right)$, then for $|\gamma| \leq m-1$ and $|x| \leq r$,

$$
\begin{aligned}
D^{\gamma} u(x)= & \int D^{\gamma} \mu(x-y) u(y) d y \\
& +\sum_{|\alpha|=m} \frac{m}{\alpha ! \sigma_{n}} \int D^{\gamma} \chi_{\alpha}(x-y) D^{\alpha} u(y) d y
\end{aligned}
$$


and

$$
\begin{aligned}
D^{\gamma} u(x)= & \int D^{\gamma} \zeta(x-y) u(y) d y \\
& +\sum_{|\alpha|=m} \frac{(-1)^{m} m !}{\alpha !} \int D^{\gamma+\alpha}\left(\xi \kappa_{2 m}\right)(x-y) D^{\alpha} u(y) d y .
\end{aligned}
$$

By Corollaries 3.3 and 3.4 we get interpolation inequalities.

Corollary 3.5. Let $r \geq 0$ and $\varepsilon>0$. Then for $u \in C^{m}\left(\mathbb{R}^{n}\right)$ and $|\gamma|<m-n / p$

$$
\max _{|x| \leq r}\left|D^{\gamma} u(x)\right| \leq C_{\varepsilon}^{1}\left(\int_{|y| \leq r+\varepsilon}|u(y)| d y+\sum_{|\alpha|=m}\left(\int_{|y| \leq r+\varepsilon}\left|D^{\alpha} u(y)\right|^{p} d y\right)^{1 / p}\right)
$$

where $C_{\varepsilon}^{1}$ is independent of $r$.

Proof. Let $|x| \leq r$ and $|\gamma|<m-n / p$. By applying Corollary 3.3 for $\varepsilon_{1}=\varepsilon / 2$ and $\varepsilon_{2}=\varepsilon$, we have

$$
\begin{aligned}
& \left|D^{\gamma} u(x)\right| \\
& \leq \int_{|x-y| \leq \varepsilon}\left|D^{\gamma} \mu(x-y) u(y)\right| d y \\
& \quad+\sum_{|\alpha|=m} \frac{m}{\alpha ! \sigma_{n}} \int_{|x-y| \leq \varepsilon}\left|D^{\gamma} \chi_{\alpha}(x-y) D^{\alpha} u(y)\right| d y \\
& \leq \max _{|y| \leq \varepsilon}\left|D^{\gamma} \mu(y)\right| \int_{|y| \leq r+\varepsilon}|u(y)| d y \\
& \quad+\sum_{|\alpha|=m} \frac{m ! \sigma_{n}}{\left(\int_{|x-y| \leq \varepsilon}\left|D^{\gamma} \chi_{\alpha}(x-y)\right|^{p^{\prime}} d y\right)^{1 / p^{\prime}}\left(\int_{|y| \leq r+\varepsilon}\left|D^{\alpha} u(y)\right|^{p} d y\right)^{1 / p}} \\
& \leq C_{\varepsilon}^{1}\left(\int_{|y| \leq r+\varepsilon}|u(y)| d y+\sum_{|\alpha|=m}\left(\int_{|y| \leq r+\varepsilon}\left|D^{\alpha} u(y)\right|^{p} d y\right)^{1 / p}\right)
\end{aligned}
$$

where

$$
\begin{aligned}
C_{\varepsilon}^{1} & =\max _{|\gamma|<m-n / p}\left(\max _{|y| \leq \varepsilon}\left|D^{\gamma} u(y)\right|+\max _{|\alpha|=m} \frac{m}{\alpha ! \sigma_{n}}\left(\int_{|y| \leq \varepsilon}\left|D^{\gamma} \chi_{\alpha}(y)\right|^{p^{\prime}} d y\right)^{1 / p^{\prime}}\right) \\
& <\infty .
\end{aligned}
$$

Corollary 3.6. Let $0<\varepsilon<1$ and $0 \leq r<1-\varepsilon$. Then for $u \in C^{m}\left(B_{1}\right)$ and $|\gamma| \leq m-1$,

$$
\max _{|x| \leq r}\left|D^{\gamma} u(x)\right| \leq C_{\varepsilon}^{2}\left(\max _{|y| \leq r+\varepsilon}|u(y)|+\sum_{|\alpha|=m} \max _{|y| \leq r+\varepsilon}\left|D^{\alpha} u(y)\right|\right)
$$

where $C_{\varepsilon}^{2}$ is independent of $r$. 
Proof. Let $|x| \leq r$ and $|\gamma| \leq m-1$. By applying Corollary 3.4 for $\varepsilon_{1}=\varepsilon / 2$ and $\varepsilon_{2}=\varepsilon$, we have

$$
\begin{aligned}
\left|D^{\gamma} u(x)\right| \leq & \int_{|x-y| \leq \varepsilon}\left|D^{\gamma} \zeta(x-y) u(y)\right| d y \\
& +\sum_{|\alpha|=m} \frac{m !}{\alpha !} \int_{|x-y| \leq \varepsilon}\left|D^{\alpha+\gamma}\left(\xi \kappa_{2 m}\right)(x-y) D^{\alpha} u(y)\right| d y \\
\leq & \max _{|y| \leq r+\varepsilon}|u(y)| \int\left|D^{\gamma} \zeta(y)\right| d y \\
& +\sum_{|\alpha|=m} \frac{m !}{\alpha !} \max _{|y| \leq r+\varepsilon}\left|D^{\alpha} u(y)\right| \int\left|D^{\alpha+\gamma}\left(\xi \kappa_{2 m}\right)(y)\right| d y \\
\leq & C_{\varepsilon}^{2}\left(\max _{|y| \leq r+\varepsilon}|u(y)|+\sum_{|\alpha|=m} \max _{|y| \leq r+\varepsilon}\left|D^{\alpha} u(y)\right|\right)
\end{aligned}
$$

where

$$
C_{\varepsilon}^{2}=\max _{|\gamma| \leq m-1}\left(\int\left|D^{\gamma} \zeta(y)\right| d y+\max _{|\alpha|=m} \frac{m !}{\alpha !} \int\left|D^{\alpha+\gamma}\left(\xi \kappa_{2 m}\right)(y)\right| d y\right)<\infty .
$$

4. The closure of $\Phi$ in spaces of Beppo Levi type. In order to define the Riesz potential of an $L^{p}$-function, we introduce the modified Riesz kernel $\kappa_{m, k}(x, y)$. For an integer $k<m$, we set

$$
\kappa_{m, k}(x, y)= \begin{cases}\kappa_{m}(x-y)-\sum_{|\gamma| \leq k} \frac{D^{\gamma} \kappa_{m}(-y)}{\gamma !} x^{\gamma}, & 0 \leq k<m, \\ \kappa_{m}(x-y), & k<0 .\end{cases}
$$

Moreover, for a locally integrable function $f$ we define

$$
U_{m, k}^{f}(x)=\int \kappa_{m, k}(x, y) f(y) d y
$$

if it exists. Concerning the existence of $U_{m, k}^{f}$, we have the following.

Lemma 4.1 (cf. [Ku: Corollary 5.9 and Proposition 5.15]). Let $f \in L^{p}$ and $k=[m-n / p]$ be the integral part of $m-n / p$.

(i) In case $m-n / p \notin \mathbb{N}, U_{m, k}^{f}$ exists and

$$
\left(\int\left|U_{m, k}^{f}(x)\right|^{p}|x|^{-m p} d x\right)^{1 / p} \leq C\|f\|_{p} .
$$

(ii) In case $m-n / p \in \mathbb{N}$, if we denote by $f_{1}$ and $f_{2}$ the restriction of $f$ 
to $\{|x|<1\}$ and to $\{|x| \geq 1\}$ respectively, then $U_{m, k-1}^{f_{1}}$ and $U_{m, k}^{f_{2}}$ exist and

$$
\begin{gathered}
\left(\int\left|U_{m, k-1}^{f_{1}}(x)\right|^{p}|x|^{-m p}(1+|\log | x||)^{-p} d x\right)^{1 / p} \leq C\left\|f_{1}\right\|_{p}, \\
\left(\int\left|U_{m, k}^{f_{2}}(x)\right|^{p}|x|^{-m p}(1+|\log | x||)^{-p} d x\right)^{1 / p} \leq C\left\|f_{2}\right\|_{p} .
\end{gathered}
$$

For a set $E \subset \mathbb{R}^{n}$, we denote by $L^{p}(E)$ the set of $L^{p}$-functions $f$ such that $f(x)=0$ on the complement of $E$.

Lemma 4.2 (cf. [Ku: Theorem 4.4]). Let $k=[m-n / p]$ and $|\alpha|=m$.

(i) In case $m-n / p \notin \mathbb{N}$, for $f \in L^{p}$,

$$
D^{\alpha} U_{m, k}^{f}=(-1)^{m} R^{\alpha} f \text {. }
$$

(ii) In case $m-n / p \in \mathbb{N}$, for $f \in L^{p}\left(B_{1}\right)$ and $g \in L^{p}\left(B_{1}^{\mathrm{c}}\right)$,

$$
D^{\alpha} U_{m, k-1}^{f}=(-1)^{m} R^{\alpha} f, \quad D^{\alpha} U_{m, k}^{g}=(-1)^{m} R^{\alpha} g .
$$

where $B_{1}^{\mathrm{c}}$ is the complement of $B_{1}$.

The Riesz potential space $R_{m}^{p}$ is defined by

$$
R_{m}^{p}= \begin{cases}\left\{U_{m, k}^{f}: f \in L^{p}\right\}, & m-n / p \notin \mathbb{N}, \\ \left\{U_{m, k-1}^{f_{1}}+U_{m, k}^{f_{2}}: f \in L^{p}, f_{1}=\left.f\right|_{B_{1}}, f_{2}=\left.f\right|_{B_{1}^{c}}\right\}, & m-n / p \in \mathbb{N},\end{cases}
$$

with $k=[m-n / p]$ where $\left.f\right|_{B_{1}}$ is the restriction of $f$ to $B_{1}$. Concerning the relation between the Riesz potential spaces and the spaces of Beppo Levi type, we have

Lemma 4.3 (cf. [Ku: Theorem 6.7]). Let $k=[m-n / p]$. Then

$$
R_{m}^{p}+\mathcal{P}_{k}= \begin{cases}\mathcal{L}_{m}^{p} \cap L^{p,-m,(\log )^{0}}, & m-n / p \notin \mathbb{N} \\ \mathcal{L}_{m}^{p} \cap L^{p,-m,(\log )^{-1}}, & m-n / p \in \mathbb{N}\end{cases}
$$

where $\mathcal{P}_{k}$ is the set of polynomials of degree $k$ with complex coefficients.

We introduce the following notation:

$$
\mathcal{B}=\left\{f \in C^{\infty}: D^{\alpha} f \text { is bounded for each } \alpha\right\} .
$$

The following lemma holds.

LemmA 4.4. (i) If $u \in \mathcal{S}$ and $v \in \mathcal{H} \cap \mathcal{B}$, then $u v \in \Psi$.

(ii) If $u \in \Psi$ and $v \in \mathcal{B}$, then $u v \in \Psi$.

We take a function $\lambda \in \mathcal{D}$ such that $\lambda(x)=1$ on $|x| \leq 1$. We put

$$
\begin{aligned}
& h(x)=\lambda(x)+e^{-|x|^{2}-1 /|x|^{2}}, \\
& g(x)=1-\lambda(x)-e^{-|x|^{2}-1 /|x|^{2}}=1-h(x) .
\end{aligned}
$$

We note that $g \in \mathcal{H} \cap \mathcal{B}$. For a function $f$ and a positive number $\varepsilon$, we set

$$
f_{(\varepsilon)}(x)=f(\varepsilon x) .
$$


We can easily check

Lemma 4.5. (i) For a multi-index $\alpha, D^{\alpha} f_{(\varepsilon)}(x)=\varepsilon^{|\alpha|} D^{\alpha} f(\varepsilon x)$.

(ii) If $f \in L^{1}$, then $\mathcal{F} f_{(\varepsilon)}(x)=\left(1 / \varepsilon^{n}\right) \mathcal{F} f(x / \varepsilon)$.

We put

$$
\nu(x)=\frac{1}{(2 \pi)^{n}} e^{-|x|^{2}}, \quad \varrho_{\varepsilon}(x)=\nu_{(\varepsilon)}(x) g_{(1 / \varepsilon)}(x)=\nu(\varepsilon x) g(x / \varepsilon) .
$$

Since $\nu_{(\varepsilon)} \in \mathcal{S}$ and $g_{(1 / \varepsilon)} \in \mathcal{H} \cap \mathcal{B}$, it follows from Lemma 2.1(i) that

$$
\varrho_{\varepsilon} \in \Psi \text {. }
$$

Further, we have

$$
\varrho_{\varepsilon}(x)=\nu_{(\varepsilon)}(x)-\nu_{(\varepsilon)}(x) h_{(1 / \varepsilon)}(x) .
$$

We set

$$
\mathcal{K}_{\varepsilon}(x)=\mathcal{F} \varrho_{\varepsilon}(x), \quad \mathcal{K}_{\varepsilon}^{1}(x)=\mathcal{F} \nu_{(\varepsilon)}(x), \quad H_{\varepsilon}(x)=\mathcal{F} h_{(\varepsilon)}(x)
$$

and

$$
\mathcal{K}_{\varepsilon}^{2}(x)=\mathcal{F}\left(\nu_{(\varepsilon)} h_{(1 / \varepsilon)}\right)(x)=\mathcal{K}_{\varepsilon}^{1} * H_{1 / \varepsilon}(x)
$$

where the symbol $*$ means convolution. Since $\varrho_{\varepsilon} \in \Psi$ by (4.1), we have $\mathcal{K}_{\varepsilon} \in \Phi$ on account of (1.2). By elementary calculations we have $\mathcal{K}_{1}^{1}(x)=$ $2^{-n} \pi^{-n / 2} e^{-|x|^{2} / 4}$, and hence

$$
\int \mathcal{K}_{1}^{1}(x) d x=1 .
$$

Moreover, by Lemma 2.2(ii) we have

$$
\mathcal{K}_{\varepsilon}^{1}(x)=\frac{1}{\varepsilon^{n}} \mathcal{K}_{1}^{1}\left(\frac{x}{\varepsilon}\right), \quad H_{1 / \varepsilon}(x)=\varepsilon^{n} H_{1}(\varepsilon x) .
$$

Lemma 4.6. If $(1+|x|)^{m} u(x) \in L^{1}$ for every nonnegative integer $m$, then $\mathcal{K}_{\varepsilon} * u \in \Phi$.

Proof. By (1.2) it suffices to show that $\overline{\mathcal{F}}\left(\mathcal{K}_{\varepsilon} * u\right) \in \Psi$. Since $\mathcal{K}_{\varepsilon} \in \mathcal{S}$ and $u \in L^{1}$, we see that

$$
\overline{\mathcal{F}}\left(\mathcal{K}_{\varepsilon} * u\right)(x)=\overline{\mathcal{F}} \mathcal{K}_{\varepsilon}(x) \overline{\mathcal{F}} u(x)=(2 \pi)^{n} \varrho_{\varepsilon}(x) \overline{\mathcal{F}} u(x)
$$

by (1.1). The condition $(1+|x|)^{m} u(x) \in L^{1} \quad(m=0,1, \ldots)$ implies $\overline{\mathcal{F}} u \in \mathcal{B}$. Therefore (4.1) and Lemma 4.4(ii) give the assertion.

We denote by $\Phi_{m}^{p}$ the closure of $\Phi$ in $\mathcal{L}_{m}^{p}$.

Lemma 4.7. Let $k=[m-n / p]$. Then $\Phi_{m}^{p} \supset \mathcal{P}_{k}$.

Proof. Let $Q \in \mathcal{P}_{k}$. We take a function $\phi \in C^{\infty}(\mathbb{R})$ such that $\phi(t)=1$ on $t \leq 1$ and $\phi(t)=0$ on $t \geq 2$. If we set 


$$
\varphi_{j}(x)= \begin{cases}\phi\left(\frac{\log |x|}{\log j}\right), & x \neq 0 \\ 1, & x=0\end{cases}
$$

then $\varphi_{j} \in \mathcal{D}$ and $\varphi_{j}(x)=1$ on $|x| \leq j$ and $\varphi_{j}(x)=0$ on $|x| \geq j^{2}$. We put $h_{j}=\varphi_{j} Q \in \mathcal{D}$. We shall show that $h_{j}$ converges to $Q$ as $j \rightarrow \infty$ in $\mathcal{L}_{m}^{p}$. It is clear that

$$
\int_{|x|<1}\left|h_{j}(x)-Q(x)\right| d x \rightarrow 0 \quad(j \rightarrow \infty) .
$$

Let $|\alpha|=m$. Since $Q \in \mathcal{P}_{k}$ and $k<m$, by the Leibniz formula we have

$$
\begin{aligned}
D^{\alpha}\left(h_{j}-Q\right)(x) & =D^{\alpha} h_{j}(x)=\sum_{\gamma \leq \alpha}\left(\begin{array}{l}
\alpha \\
\gamma
\end{array}\right) D^{\alpha-\gamma} \varphi_{j}(x) D^{\gamma} Q(x) \\
& =\sum_{\gamma \leq \alpha,|\gamma| \leq k}\left(\begin{array}{l}
\alpha \\
\gamma
\end{array}\right) D^{\alpha-\gamma} \varphi_{j}(x) D^{\gamma} Q(x) .
\end{aligned}
$$

Since $\alpha-\gamma \neq 0$ for $|\gamma| \leq k$, we see that

$$
\begin{aligned}
& \left(\int\left|D^{\alpha}\left(h_{j}-Q\right)(x)\right|^{p} d x\right)^{1 / p} \\
& \leq \sum_{\gamma \leq \alpha,|\gamma| \leq k}\left(\begin{array}{l}
\alpha \\
\gamma
\end{array}\right)\left(\int_{j \leq|x| \leq j^{2}}\left|D^{\alpha-\gamma} \varphi_{j}(x) D^{\gamma} Q(x)\right|^{p} d x\right)^{1 / p} \\
& \leq C \sum_{\gamma \leq \alpha,|\gamma| \leq k}\left(\int_{j \leq|x| \leq j^{2}}\left|D^{\alpha-\gamma} \varphi_{j}(x)\right|^{p}|x|^{(k-|\gamma|) p} d x\right)^{1 / p} .
\end{aligned}
$$

Since

$$
\left|D^{\alpha-\gamma} \varphi_{j}(x)\right| \leq \frac{C}{(\log j)|x|^{|\alpha-\gamma|}}
$$

on $j \leq|x| \leq j^{2}$, we obtain

$$
\begin{aligned}
\int_{j \leq|x| \leq j^{2}}\left|D^{\alpha-\gamma} \varphi_{j}(x)\right|^{p}|x|^{(k-|\gamma|) p} d x & \\
& \leq \frac{C}{(\log j)^{p}} \begin{cases}\int_{j \leq|x|}|x|^{(k-m) p} d x, \quad k<m-n / p \\
\int_{j \leq|x| \leq j^{2}}|x|^{-n} d x, \quad k=m-n / p\end{cases} \\
& =\frac{C}{(\log j)^{p}} \begin{cases}j^{(k-m) p+n}, & k<m-n / p \\
\log j, & k=m-n / p\end{cases} \\
& \rightarrow 0 \quad(j \rightarrow \infty)
\end{aligned}
$$


since $(k-m) p+n<0$ for $k<m-n / p$. Thus we get

$$
\left\|h_{j}-Q\right\|_{\mathcal{L}_{m}^{p}} \rightarrow 0 \quad(j \rightarrow \infty) .
$$

Further, we put $h_{j, \varepsilon}=\mathcal{K}_{\varepsilon} * h_{j}=\mathcal{K}_{\varepsilon}^{1} * h_{j}-\mathcal{K}_{\varepsilon}^{2} * h_{j}$. Since $h_{j} \in \mathcal{D},(4.2)$ and (4.3) imply

$$
\left\|\mathcal{K}_{\varepsilon}^{1} * h_{j}-h_{j}\right\|_{\mathcal{L}_{m}^{p}} \rightarrow 0 \quad(\varepsilon \rightarrow 0) .
$$

Since $\mathcal{K}_{\varepsilon}^{2} * h_{j}=\mathcal{K}_{\varepsilon}^{1} * H_{1 / \varepsilon} * h_{j}$, we obtain

$$
\left\|\mathcal{K}_{\varepsilon}^{2} * h_{j}\right\|_{\mathcal{L}_{m}^{p}} \rightarrow 0 \quad(\varepsilon \rightarrow 0)
$$

because $\left\|\mathcal{K}_{\varepsilon}^{1}\right\|_{1}=1$ and $\left\|H_{1 / \varepsilon}\right\|_{p} \rightarrow 0(\varepsilon \rightarrow 0)$. Since $h_{j, \varepsilon} \in \Phi$ by Lemma 4.6, (4.4)-(4.6) give the lemma.

Now we are in a position to prove our main theorem.

THEOREM 4.8 .

$$
\Phi_{m}^{p}= \begin{cases}\mathcal{L}_{m}^{p} \cap L^{p,-m,(\log )^{0}}, & m-n / p \notin \mathbb{N}, \\ \mathcal{L}_{m}^{p} \cap L^{p,-m,(\log )^{-1}}, & m-n / p \in \mathbb{N} .\end{cases}
$$

Proof. We begin with the proof that the left-hand side is contained in the right-hand side. Let $u \in \Phi_{m}^{p}$. By the definition there exists a sequence $\left\{u_{j}\right\} \subset \Phi$ which converges to $u$ as $j \rightarrow \infty$ in $\mathcal{L}_{m}^{p}$. We put $f=D^{m} u$ and $f_{j}=D^{m} u_{j}(j=1,2, \ldots)$. It follows from $u \in \mathcal{L}_{m}^{p}$ that $f \in L^{p}$, and by Corollary $2.8, f_{j} \in \Phi$ and $u_{j}=\kappa_{m}^{f_{j}}$. Moreover, we see that

$$
\begin{aligned}
\left\|f_{j}-f\right\|_{p} & \leq \sum_{|\alpha|=m} \frac{m !}{\alpha !}\left\|R^{\alpha} D^{\alpha}\left(u_{j}-u\right)\right\|_{p} \\
& \leq C\left|u_{j}-u\right|_{m, p} \rightarrow 0 \quad(j \rightarrow \infty) .
\end{aligned}
$$

Let $k=[m-n / p]$. We first consider the case $m-n / p \notin \mathbb{N}$. We have

$$
u_{j}(x)=\int \kappa_{m}(x-y) f_{j}(y) d y=U_{m, k}^{f_{j}}(x)+\sum_{|\gamma| \leq k} a_{\gamma, j} x^{\gamma}
$$

with

$$
a_{\gamma, j}=\frac{1}{\gamma !} \int D^{\gamma} \kappa_{m}(-y) f_{j}(y) d y=\frac{D^{\gamma} u_{j}(0)}{\gamma !} .
$$

Lemma 4.1(i) and (4.7) give

$$
\int_{|x|<1}\left|U_{m, k}^{f_{j}}(x)-U_{m, k}^{f}(x)\right| d x \rightarrow 0 \quad(j \rightarrow \infty) .
$$

Further, for $|\alpha|=m$, by Lemma 4.2(i), (2.4) and (4.7) we see that

$$
\left\|D^{\alpha}\left(U_{m, k}^{f_{j}}-U_{m, k}^{f}\right)\right\|_{p}=C\left\|R^{\alpha} f_{j}-R^{\alpha} f\right\|_{p} \leq C\left\|f_{j}-f\right\|_{p} \rightarrow 0 \quad(j \rightarrow \infty) .
$$


Thus we conclude that $U_{m, k}^{f_{j}}$ converges to $U_{m, k}^{f}$ as $j \rightarrow \infty$ in $\mathcal{L}_{m}^{p}$. For $|\gamma| \leq k$, by applying Corollary 3.5 with $r=0$ and $\varepsilon=1$, we have

$$
\begin{aligned}
& \left|a_{\gamma, j}-a_{\gamma, l}\right|=\frac{1}{\gamma !}\left|D^{\gamma} u_{j}(0)-D^{\gamma} u_{l}(0)\right| \\
& \leq C\left(\int_{|y| \leq 1}\left|u_{j}(y)-u_{l}(y)\right| d y+\sum_{|\alpha|=m}\left(\int_{|y| \leq 1}\left|D^{\alpha} u_{j}(y)-D^{\alpha} u_{l}(y)\right|^{p} d y\right)^{1 / p}\right) \\
& \leq C\left\|u_{j}-u_{l}\right\|_{\mathcal{L}_{m}^{p}} \rightarrow 0 \quad(j, l \rightarrow \infty) .
\end{aligned}
$$

Thus $\left\{a_{\gamma, j}\right\}_{j=1}^{\infty}$ is a Cauchy sequence for each $|\gamma| \leq k$, and hence there exist $a_{\gamma}(|\gamma| \leq k)$ such that $a_{\gamma, j}$ tends to $a_{\gamma}$ as $j \rightarrow \infty$. This implies that $\sum_{|\gamma| \leq k} a_{\gamma, j} x^{\gamma}$ converges to $\sum_{|\gamma| \leq k} a_{\gamma} x^{\gamma}$ as $j \rightarrow \infty$ in $\mathcal{L}_{m}^{p}$. Consequently, we have

$$
u=U_{m, k}^{f}+\sum_{|\gamma| \leq k} a_{\gamma} x^{\gamma} \in R_{m}^{p}+\mathcal{P}_{k}
$$

This implies that $u \in \mathcal{L}_{m}^{p} \cap L^{p,-m,(\log )^{0}}$ by Lemma 4.3 .

We next consider the case $m-n / p \in \mathbb{N}$. In this case, putting $f_{j, 1}=\left.f_{j}\right|_{B_{1}}$ and $f_{j, 2}=\left.f_{j}\right|_{B_{1}^{c}}$, we have

$$
u_{j}(x)=\int \kappa_{m}(x-y) f_{j}(y) d y=U_{m, k-1}^{f_{j, 1}}(x)+U_{m, k}^{f_{j, 2}}(x)+\sum_{|\gamma| \leq k} a_{\gamma, j} x^{\gamma}
$$

with

$$
a_{\gamma, j}= \begin{cases}\frac{1}{\gamma !} \int D^{\gamma} \kappa_{m}(-y) f_{j}(y) d y, & |\gamma| \leq k-1, \\ \frac{1}{\gamma !} \int_{|y| \geq 1} D^{\gamma} \kappa_{m}(-y) f_{j}(y) d y, & |\gamma|=k .\end{cases}
$$

Lemma 4.1(ii) and (4.7) give

$$
\int_{|x|<1}\left|U_{m, k-1}^{f_{j, 1}}(x)-U_{m, k-1}^{f_{1}}(x)\right| d x \rightarrow 0 \quad(j \rightarrow \infty),
$$

and

$$
\int_{|x|<1}\left|U_{m, k}^{f_{j, 2}}(x)-U_{m, k}^{f_{2}}(x)\right| d x \rightarrow 0 \quad(j \rightarrow \infty)
$$

where $f_{1}=\left.f\right|_{B_{1}}$ and $f_{2}=\left.f\right|_{B_{1}^{c}}$. Further, Lemma 4.2(ii), (2.4) and (4.7) give

$$
\left|U_{m, k-1}^{f_{j, 1}}-U_{m, k-1}^{f_{1}}\right|_{m, p} \rightarrow 0, \quad\left|U_{m, k}^{f_{j, 2}}-U_{m, k}^{f_{2}}\right|_{m, p} \rightarrow 0 \quad(j \rightarrow \infty) .
$$

Thus $U_{m, k-1}^{f_{j, 1}}+U_{m, k}^{f_{j, 2}}$ converges to $U_{m, k-1}^{f_{1}}+U_{m, k}^{f_{2}}$ as $j \rightarrow \infty$ in $\mathcal{L}_{m}^{p}$. For $|\gamma| \leq k$, we shall show that $\left\{a_{\gamma, j}\right\}_{j=1}^{\infty}$ is a Cauchy sequence. First let $m-n / p \in \mathbb{N}$ 
and $m-n / p \neq 0$. For $|\gamma| \leq k-1$, by using Corollary 3.5 we have

$$
\left|a_{\gamma, j}-a_{\gamma, l}\right|=\frac{1}{\gamma !}\left|D^{\gamma} u_{j}(0)-D^{\gamma} u_{l}(0)\right| \leq C\left\|u_{j}-u_{l}\right\|_{\mathcal{L}_{m}^{p}}
$$

because $|\gamma|<m-n / p$. This shows that $\left\{a_{\gamma, j}\right\}_{j=1}^{\infty}$ is a Cauchy sequence for $|\gamma| \leq k-1$. Let $|\gamma|=k$. We put

$$
v_{j}(x)=\int_{|y|<1} \kappa_{m}(x-y) f_{j}(y) d y, \quad w_{j}(x)=\int_{|y| \geq 1} \kappa_{m}(x-y) f_{j}(y) d y .
$$

We show that $\left\{v_{j}\right\}_{j=1}^{\infty}$ and $\left\{w_{j}\right\}_{j=1}^{\infty}$ are Cauchy sequences in $\mathcal{L}_{m}^{p}$. Since

$$
v_{j}(x)=U_{m, k-1}^{f_{j, 1}}(x)+\sum_{|\beta| \leq k-1} \frac{x^{\beta}}{\beta !} \int_{|y|<1} D^{\beta} \kappa_{m}(-y) f_{j}(y) d y,
$$

for $|\alpha|=m$ by Lemma 4.2(ii) we have

$$
D^{\alpha} v_{j}=D^{\alpha} U_{m, k-1}^{f_{j, 1}}=(-1)^{m} R^{\alpha} f_{j, 1} .
$$

Hence we see that

$$
\begin{aligned}
\left|v_{j}-v_{l}\right|_{m, p} & =\sum_{|\alpha|=m}\left\|R^{\alpha} f_{j, 1}-R^{\alpha} f_{l, 1}\right\|_{p} \leq C\left\|f_{j, 1}-f_{l, 1}\right\|_{p} \\
& \leq C\left\|f_{j}-f_{l}\right\|_{p} \rightarrow 0 \quad(j, l \rightarrow \infty)
\end{aligned}
$$

Further we obtain

$$
\begin{aligned}
\int_{|x|<1}\left|v_{j}(x)-v_{l}(x)\right| d x & =\int_{|x|<1} \mid \int_{|y|<1} \kappa_{m}(x-y)\left(f_{j}(y)-f_{l}(y) d y \mid d x\right. \\
& \leq \int_{|x|<2}\left|\kappa_{m}(x)\right| d x \int_{|y|<1}\left|f_{j}(y)-f_{l}(y)\right| d y \\
& \leq C\left\|f_{j}-f_{l}\right\|_{p} \rightarrow 0 \quad(j, l \rightarrow \infty) .
\end{aligned}
$$

Thus $\left\{v_{j}\right\}_{j=1}^{\infty}$ is a Cauchy sequence in $\mathcal{L}_{m}^{p}$. Since $u_{j}=v_{j}+w_{j}$ and $\left\{u_{j}\right\}_{j=1}^{\infty},\left\{v_{j}\right\}_{j=1}^{\infty}$ are Cauchy sequences in $\mathcal{L}_{m}^{p}$, so is $\left\{w_{j}\right\}_{j=1}^{\infty}$. Furthermore we shall show

$$
\begin{gathered}
\max _{|x| \leq 1 / 2}\left|w_{j}(x)-w_{l}(x)\right| \leq C\left\|w_{j}-w_{l}\right\|_{\mathcal{L}_{m}^{p}}, \\
\sum_{|\alpha|=m} \max _{|x| \leq 1 / 2}\left|D^{\alpha} w_{j}(x)-D^{\alpha} w_{l}(x)\right| \leq C\left\|w_{j}-w_{l}\right\|_{\mathcal{L}_{m}^{p}} .
\end{gathered}
$$

Since $w_{j}-w_{l} \in C^{\infty}\left(B_{1}\right)$, by applying Corollary 3.4 with $r=1 / 2,0<\varepsilon_{1}<$ $\varepsilon_{2}<1 / 2$ and $\gamma=0$, for $|x| \leq 1 / 2$ we have 


$$
\begin{aligned}
& \left|w_{j}(x)-w_{l}(x)\right| \\
& \leq\left|\int_{|x-y| \leq \varepsilon_{2}} \zeta(x-y)\left(w_{j}(y)-w_{l}(y)\right) d y\right| \\
& \quad+\sum_{|\alpha|=m} \frac{m !}{\alpha !}\left|\int_{|x-y| \leq \varepsilon_{2}} D^{\alpha}\left(\xi \kappa_{2 m}\right)(x-y) D^{\alpha}\left(w_{j}-w_{l}\right)(y) d y\right| \\
& \leq\|\zeta\|_{\infty} \int_{|y|<1}\left|w_{j}(y)-w_{l}(y)\right| d y \\
& \quad+\sum_{|\alpha|=m} \frac{m !}{\alpha !}\left(\int_{|y|<1}\left|D^{\alpha}\left(\xi \kappa_{2 m}\right)(y)\right|^{p^{\prime}} d y\right)^{1 / p^{\prime}}\left(\int_{|y|<1}\left|D^{\alpha}\left(w_{j}-w_{l}\right)(y)\right|^{p} d y\right)^{1 / p} \\
& \leq C\left\|w_{j}-w_{l}\right\|_{\mathcal{L}_{m}^{p}}^{p}
\end{aligned}
$$

with $\|\zeta\|_{\infty}=\sup _{x}|\zeta(x)|$, because $m-n / p>0$ implies

$$
\left(\int_{|y|<1}\left|D^{\alpha}\left(\xi \kappa_{2 m}\right)(y)\right|^{p^{\prime}} d y\right)^{1 / p^{\prime}}<\infty
$$

Thus we obtain (4.8). Further, for $|\alpha|=m$ and $|x| \leq 1 / 2$ we have

$$
\begin{aligned}
\mid D^{\alpha} w_{j} & (x)-D^{\alpha} w_{l}(x)|=| \int_{|y| \geq 1} D^{\alpha} \kappa_{m}(x-y)\left(f_{j}(y)-f_{l}(y)\right) d y \mid \\
& \leq\left(\int_{|y| \geq 1}\left|D^{\alpha} \kappa_{m}(x-y)\right|^{p^{\prime}} d y\right)^{1 / p^{\prime}}\left(\int_{|y| \geq 1}\left|f_{j}(y)-f_{l}(y)\right|^{p} d y\right)^{1 / p} \\
& \leq\left(\int_{|y| \geq 1 / 2}\left|D^{\alpha} \kappa_{m}(y)\right|^{p^{\prime}} d y\right)^{1 / p^{\prime}}\left(\int_{|y| \geq 1}\left|f_{j}(y)-f_{l}(y)\right|^{p} d y\right)^{1 / p} \\
& \leq C\left\|f_{j, 2}-f_{l, 2}\right\|_{p}=C\left\|\sum_{|\gamma|=m} \frac{m !}{\gamma !} R^{\gamma} D^{\gamma}\left(w_{j}-w_{l}\right)\right\|_{p} \\
& \leq C\left|w_{j}-w_{l}\right|_{m, p} \leq C\left\|w_{j}-w_{l}\right\| \mathcal{L}_{m}^{p}
\end{aligned}
$$

because

$$
\left(\int_{|y| \geq 1 / 2}\left|D^{\alpha} \kappa_{m}(y)\right|^{p^{\prime}} d y\right)^{1 / p^{\prime}} \leq C\left(\int_{|y| \geq 1 / 2}|y|^{-n p^{\prime}} d y\right)^{1 / p^{\prime}}<\infty .
$$

Thus we obtain (4.9). Consequently, by taking $|\gamma|=k, r=0$ and $\varepsilon=1 / 2$ in Corollary 3.6, we obtain

$$
\left|a_{\gamma, j}-a_{\gamma, l}\right|=\frac{1}{\gamma !}\left|D^{\gamma} w_{j}(0)-D^{\gamma} w_{l}(0)\right| \leq C\left\|w_{j}-w_{l}\right\|_{\mathcal{L}_{m}^{p}} .
$$


This shows that $\left\{a_{\gamma, j}\right\}_{j=1}^{\infty}$ is a Cauchy sequence for $|\gamma|=k$. Next let $m-n / p=0$. In this case we have

$$
u_{j}(x)=\kappa_{m}^{f_{j}}(x)=U_{m,-1}^{f_{j, 1}}(x)+U_{m, 0}^{f_{j, 2}}(x)+a_{0, j} .
$$

By putting $\omega_{n}=\int_{|x|<1} d x$, we see that

$$
\begin{aligned}
& \left|a_{0, j}-a_{0, l}\right|=\frac{1}{\omega_{n}} \int_{|x|<1}\left|a_{0, j}-a_{0, l}\right| d x \\
& =\frac{1}{\omega_{n}} \int_{|x|<1}\left|u_{j}(x)-u_{l}(x)+U_{m,-1}^{f_{l, 1}}(x)-U_{m,-1}^{f_{j, 1}}(x)+U_{m, 0}^{f_{l, 2}}(x)-U_{m, 0}^{f_{j, 2}}(x)\right| d x \\
& \leq \frac{1}{\omega_{n}} \int_{|x|<1}\left|u_{j}(x)-u_{l}(x)\right| d x+\omega_{n}^{-1 / p}\left(\int_{|x|<1}\left|U_{m,-1}^{f_{l, 1}-f_{j, 1}}(x)\right|^{p} d x\right)^{1 / p} \\
& \quad+\omega_{n}^{-1 / p}\left(\int_{|x|<1}\left|U_{m, 0}^{f_{l, 2}-f_{j, 2}}(x)\right|^{p} d x\right)^{1 / p} .
\end{aligned}
$$

Therefore, Lemma 4.1(ii) gives

$$
\begin{aligned}
\left|a_{0, j}-a_{0, l}\right| & \leq C\left\|u_{j}-u_{l}\right\|_{\mathcal{L}_{m}^{p}}+C\left\|f_{l, 1}-f_{j, 1}\right\|_{p}+C\left\|f_{l, 2}-f_{j, 2}\right\|_{p} \\
& \leq C\left\|u_{j}-u_{l}\right\|_{\mathcal{L}_{m}^{p}}+C\left\|f_{l}-f_{j}\right\|_{p} \leq C\left\|u_{j}-u_{l}\right\|_{\mathcal{L}_{m}^{p}} .
\end{aligned}
$$

This shows that $\left\{a_{0, j}\right\}_{j=1}^{\infty}$ is a Cauchy sequence. Thus we conclude that $\left\{a_{\gamma, j}\right\}_{j=1}^{\infty}$ is a Cauchy sequence for $|\gamma| \leq k$. Hence there exist $a_{\gamma}(|\gamma| \leq k)$ such that $a_{\gamma, j}$ tends to $a_{\gamma}$ as $j \rightarrow \infty$. This implies that $\sum_{|\gamma| \leq k} a_{\gamma, j} x^{\gamma}$ converges to $\sum_{|\gamma| \leq k} a_{\gamma} x^{\gamma}$ as $j \rightarrow \infty$ in $\mathcal{L}_{m}^{p}$. Consequently, we have

$$
u=U_{m, k-1}^{f_{1}}+U_{m, k}^{f_{2}}+\sum_{|\gamma| \leq k} a_{\gamma} x^{\gamma} \in R_{m}^{p}+\mathcal{P}_{k} .
$$

This implies that $u \in \mathcal{L}_{m}^{p} \cap L^{p,-m,(\log )^{-1}}$ by Lemma 4.3.

Next, we show the converse inclusion. Let

$$
u \in \begin{cases}\mathcal{L}_{m}^{p} \cap L^{p,-m,(\log )^{0}}, & m-n / p \notin \mathbb{N}, \\ \mathcal{L}_{m}^{p} \cap L^{p,-m,(\log )^{-1}}, & m-n / p \in \mathbb{N} .\end{cases}
$$

By Lemma 4.3 there exists an $L^{p}$-function $f$ such that

$$
u= \begin{cases}U_{m, k}^{f}+\sum_{|\gamma| \leq k} a_{\gamma} x^{\gamma}, & m-n / p \notin \mathbb{N}, \\ U_{m, k-1}^{f_{1}}+U_{m, k}^{f_{2}}+\sum_{|\gamma| \leq k} a_{\gamma} x^{\gamma}, & m-n / p \in \mathbb{N},\end{cases}
$$

with $k=[m-n / p], f_{1}=\left.f\right|_{B_{1}}$ and $f_{2}=\left.f\right|_{B_{1}^{c}}$. Since $\Phi$ is dense in $L^{p}([\operatorname{Li} 1])$, there exists a sequence $\left\{f_{j}\right\} \subset \Phi$ such that $f_{j}$ converges to $f$ as $j \rightarrow \infty$ in $L^{p}$. We consider the case $m-n / p \notin \mathbb{N}$. Since $f_{j}$ converges to $f$ as $j \rightarrow \infty$ in $L^{p}, U_{m, k}^{f_{j}}$ converges to $U_{m, k}^{f}$ as $j \rightarrow \infty$ in $\mathcal{L}_{m}^{p}$ by Lemmas 4.1(i) and 4.2(i). 
Hence, for given $\varepsilon>0$, there exists a function $f_{l} \in \Phi$ such that

$$
\begin{aligned}
\varepsilon / 2 & >\left\|U_{m, k}^{f}-U_{m, k}^{f_{l}}\right\|_{\mathcal{L}_{m}^{p}} \\
& =\left\|U_{m, k}^{f}-\kappa_{m}^{f_{l}}+\sum_{|\gamma| \leq k} \frac{x^{\gamma}}{\gamma !} \int D^{\gamma} \kappa_{m}(-y) f_{l}(y) d y\right\|_{\mathcal{L}_{m}^{p}} .
\end{aligned}
$$

We put

$$
P(x)=\sum_{|\gamma| \leq k} a_{\gamma} x^{\gamma}, \quad P_{l}(x)=\sum_{|\gamma| \leq k} \frac{x^{\gamma}}{\gamma !} \int D^{\gamma} \kappa_{m}(-y) f_{l}(y) d y .
$$

Since $P-P_{l} \in \mathcal{P}_{k}$, by Lemma 4.7 there exists a function $\varphi \in \Phi$ such that

$$
\left\|P-P_{l}-\varphi\right\|_{\mathcal{L}_{m}^{p}}<\varepsilon / 2
$$

By (4.10) and (4.11) we have

$$
\begin{aligned}
\left\|u-\kappa_{m}^{f_{l}}-\varphi\right\|_{\mathcal{L}_{m}^{p}} & =\left\|U_{m, k}^{f}+P+P_{l}-P_{l}-\kappa_{m}^{f_{l}}-\varphi\right\|_{\mathcal{L}_{m}^{p}} \\
& \leq\left\|U_{m, k}^{f}-\kappa_{m}^{f_{l}}+P_{l}\right\|_{\mathcal{L}_{m}^{p}}+\left\|P-P_{l}-\varphi\right\|_{\mathcal{L}_{m}^{p}} \\
& =\left\|U_{m, k}^{f}-U_{m, k}^{f_{l}}\right\|_{\mathcal{L}_{m}^{p}}+\left\|P-P_{l}-\varphi\right\|_{\mathcal{L}_{m}^{p}} \\
& <\varepsilon / 2+\varepsilon / 2=\varepsilon .
\end{aligned}
$$

Since $\kappa_{m}^{f_{l}}+\varphi \in \Phi$ by Corollary 2.6, this implies that $u \in \Phi_{m}^{p}$. In case $m-n / p \in \mathbb{N}$, we can prove that $u \in \Phi_{m}^{p}$ in the same way. Thus we have completed the proof of the theorem.

Remark 4.9. Since $\mathcal{L}_{m}^{p}=R_{m}^{p}+\mathcal{P}_{m-1}([\mathrm{Ku}$ : Theorems 6.1 and 6.3$])$, by Lemma 4.3 and Theorem 4.8 we conclude that if $p \geq n$, then $\Phi$ is dense in $\mathcal{L}_{m}^{p}$, and if $p<n$, then $\Phi$ is not dense in $\mathcal{L}_{m}^{p}$.

\section{References}

[He] S. Helgason, The Radon Transform, Birkhäuser, Boston, MA, 1980.

[Ku] T. Kurokawa, Riesz potentials, higher Riesz transforms and Beppo Levi spaces, Hiroshima Math. J. 18 (1988), 541-597.

[Li1] P. I. Lizorkin, Generalized Liouville differentiation and the multiplier method in the theory of imbedding of classes of differentiable functions, Proc. Steklov Inst. Math. 105 (1969), 105-202.

[Li2] - Operators connected with fractional differentiation and classes of differentiable functions, ibid. 117 (1972), 251-286.

[Re] Yu. G. Reshetnyak, The concept of capacity in the theory of functions with generalized derivatives, Siberian Math. J. 10 (1969), 818-842.

[Sa] S. G. Samko, Denseness of the Lizorkin-type spaces $\Phi_{V}$ in $L_{p}\left(\mathbb{R}^{n}\right)$, Math. Notes 31 (1982), no. 6, 432-437.

[SKM] S. G. Samko, A. A. Kilbas and O. I. Marichev, Fractional Integrals and Derivatives, Gordon and Breach, 1993. 
[Sc] L. Schwartz, Théorie des distributions, Hermann, Paris, 1966.

[Wa] H. Wallin, Continuous functions and potential theory, Ark. Mat. 5 (1963), 55-84.

Department of Mathematics

and Computer Science

Kagoshima University

890-0065 Kagoshima, Japan

E-mail: kurokawa@sci.kagoshima-u.ac.jp 